\title{
Identification of metabolomic changes in horse plasma after racing by liquid chromatography-high resolution mass spectrometry as a strategy for doping testing
}

\author{
Toshiki UEDA $^{1}$, Teruaki TOZAKI ${ }^{2 *}$, Satoshi NOZAWA ${ }^{1}$, Kenji KINOSHITA ${ }^{1}$ and \\ Hitoshi GAWAHARA ${ }^{1}$ \\ ${ }^{1}$ Drug Analysis Department, Laboratory of Racing Chemistry, Tochigi 320-0851, Japan \\ ${ }^{2}$ Genetic Analysis Department, Laboratory of Racing Chemistry, Tochigi 320-0851, Japan
}

Recently, the illegal use of novel technologies, such as gene and cell therapies, has become a great concern for the horseracing industry. As a potential way to control this, metabolomics approaches that comprehensively analyze metabolites in biological samples have been gaining attention. However, it may be difficult to identify metabolic biomarkers for doping because physiological conditions generally differ between resting and exercise states in horses. To understand the metabolic differences in horse plasma between the resting state at training centres and the sample collection stage after racing for doping test $(S A D)$, we took plasma samples from these two stages ( $n=30$ for each stage) and compared the metabolites present in these samples by liquid chromatography-high resolution mass spectrometry. This analysis identified 5,010 peaks, of which 1,256 peaks (approximately $25 \%)$ were annotated using KEGG analysis. Principal component analysis showed that the resting state and SAD groups had entirely different metabolite compositions. In particular, the levels of inosine, xanthosine, uric acid, and allantoin, which are induced by extensive exercise, were significantly increased in the SAD group. In addition, many metabolites not affected by extensive exercise were also identified. These results will contribute to the discovery of biomarkers for detecting doping substances that cannot be detected by conventional methods.

Key words: doping control, gene and cell doping, horse racing, liquid chromatographyhigh resolution mass spectrometry, metabolomics

Thoroughbred horse racing began in the 17 th century in England and is currently conducted in over sixty countries. As a sport that is heavily gambled on, horse racing should be fairly managed, and controlling doping is one of the primary concerns for horse racing authorities. In addition, anti-doping in horse racing contributes to the selection of superior individuals.

At present, low molecular weight compounds, such as caffeine and furosemide, are the main prohibited substances

Received: May 29, 2019

Accepted: August 1, 2019

*Corresponding author. e-mail: ttozaki@1rc.or.jp

C2019 Japanese Society of Equine Science

This is an open-access article distributed under the terms of the Creative Commons Attribution Non-Commercial No Derivatives (by-nc-nd) License. (CC-BY-NC-ND 4.0: https://creativecommons.org/licenses/ by-nc-nd/4.0/)

\author{
J. Equine Sci. \\ Vol. 30, No. 3 \\ pp. 55-61, 2019
}

that are routinely checked in doping tests in horses. Mass spectrometry (MS) directly detects these substances [5, 15] as well as peptides and heavy metals, such as cobalt $[4,6$, $7,23]$. However, these methods can only detect compounds with known molecular weights and structures; therefore, unknown designer drugs cannot be detected. In addition, with the rapid development of medical technologies such as gene and cell therapies, illegal uses of new medical technologies that are defined as gene and cell doping are becoming a major concern for the horse racing industry. According to the agreement of the International Federation of Horseracing Authorities (IFHA), the owner or trainer must maintain full and accurate records of all such therapies. While direct detection methods based on the polymerase chain reaction (PCR) amplification of exogenous genes have been developed [20,21], they require certain genes to be identified as markers against which primer and probe 
sequences have to be designed. In short, mass spectrometry and PCR cannot directly detect doping substances without prior information.

Metabolomic analysis is a method that comprehensively detects and analyses metabolites in the body; therefore, metabolomics approaches could potentially be used for indirectly detecting some unknown doping substances. In general, metabolomics targets low molecular weight biological compounds $(<1,000 \mathrm{Da})$, such as amino acids, nucleotides, lipids, and sugar. Metabolomics approaches are currently used in several medical $[3,9,17]$ and food fields $[8,11,12]$. Several metabolomics studies have been reported in humans and horses. Hypoxanthine was identified as a candidate biomarker for detecting salbutamol in humans [22]. Similarly, 1-cyclopentenoylglycine may be a new biomarker of testosterone misuse [16]. In horses, several biomarkers have been identified to detect the misuse of the erythropoiesis receptor activator [10].

However, there are some concerns about using metabolomics approaches for the detection of doping substances. When applying metabolomics approaches, animals need to be maintained under controlled conditions because biological components often reflect their physiological states. In order to identify biomarkers for detecting the use of prohibited substances, samples are normally obtained under resting conditions. In Japan, samples (urine and/or blood) for testing are taken within an hour of a race finishing. While one hour may be sufficient for the racehorse to recover from extensive exercise, biological components metabolized as a result of this exercise may not fully recover until a complete return to resting/normal conditions. Because of this, experimentally discovered doping biomarkers cannot be used for routine doping testing.

We hypothesized that several biological components in plasma are quantitatively and qualitatively different between the resting state and the stage after racing for doping test (SAD). Therefore, we investigated the metabolic differences between these two states by comprehensively comparing metabolites using liquid chromatography-high resolution mass spectrometry (LC-HRMS) to explore metabolites which might or might not be affected by exercise. LC-HRMS is a widely used technique in metabolomics. We used LC-HRMS method for humans [2] because metabolites are common between animal species.

\section{Materials and Methods}

\section{Ethics and sample collection}

All plasma samples used in this study were provided by the Japan Racing Association (JRA). The JRA approved the use of all provided samples for this study. In this study, blood samples were collected from thoroughbreds at SAD and from thoroughbreds under resting conditions at JRA training centres. Blood samples were centrifuged to isolate the plasma. Plasma samples were stored at $-30^{\circ} \mathrm{C}$. From the provided samples, 30 samples for $\mathrm{SAD}$ and resting states were randomly selected for this study.

\section{Sample preparation}

Deproteinization was carried out according to the method described by Dunn et al. [2]. Plasma $(100 \mu l)$ was diluted with methanol $(300 \mu l)$, briefly mixed, and centrifuged at $15,800 \times g$ at room temperature for $15 \mathrm{~min}$. The supernatant was dried in a centrifugal vacuum evaporator for 18 $\mathrm{hr}$, dissolved in $100 \mu \mathrm{l}$ ultrapure water, and centrifuged at $15,800 \times g$ at room temperature for $15 \mathrm{~min}$. Finally, $90 \mu \mathrm{l}$ of the dissolved sample was transferred to a vial. A quality control (QC) sample and phosphate buffered saline were prepared as control and blank samples, respectively, using the same protocol.

\section{LC-HRMS analysis}

For chromatographic separation, a Thermo Fisher Scientific Dionex Ultimate 3000 high performance liquid chromatography system (Thermo Fisher Scientific, Waltham, MA, U.S.A.) was used. Separation was carried out on an ACQUITY BEH C18 column $(100 \mathrm{~mm} \times 2.1 \mathrm{~mm}, 1.7 \mu \mathrm{m})$. The column oven was set at $50^{\circ} \mathrm{C}$ and a flow rate of $0.3 \mathrm{ml} /$ min was used. Solvent A was composed of $0.1 \%$ formic acid in water, and solvent $\mathrm{B}$ was composed of $0.1 \%$ formic acid in methanol. Metabolites were eluted using the following gradient elution method: from $0 \% \mathrm{~B}$ at $1 \mathrm{~min}$ to $100 \% \mathrm{~B}$ in $15 \mathrm{~min}$, held for $2 \mathrm{~min}$, returned to the initial conditions in $2 \mathrm{~min}$, and then held for $2 \mathrm{~min}$. The total run time was 22 $\min$. The injection volume was $5 \mu l$.

Detection was performed using a Q Exactive HF Quadrupole-Orbitrap mass spectrometer (Thermo Fisher Scientific) operated in a polarity switching mode. The ion source conditions were as follows: sheath gas flow rate, auxiliary gas flow rate, and sweep gas flow rate $=50,20$, and 0 (arbitrary units), respectively; capillary temperature $=250^{\circ} \mathrm{C}$; heater temperature $=400^{\circ} \mathrm{C}$; spray voltage $=+3.00 \mathrm{kV}$ (positive ionization mode) and $-3.00 \mathrm{kV}$ (negative ionization mode); lock mass $=$ off. Nitrogen was used as both the source and the collision gas. Data acquisition was performed at a resolution of 60,000 (FWHM). Each acquisition cycle included a full scan in positive ionization acquisition mode and a full-scan in negative ionization acquisition mode. The automatic gain control (AGC) target was set to $1 \mathrm{e}^{6}$, and the scan range was $m / z 100-1,000$ for all acquisition events. For identification of metabolites, MS/MS spectra were obtained by data-dependent MS/MS (ddMS ${ }^{2}$ ) and parallel reaction monitoring (PRM) mode, and the following parameters were used in $\mathrm{ddMS}^{2}$ and PRM: resolution=15,000; normal- 
ized collision energy $=30,80$, or 100 ; isolation window $=2.0$ or 1.4; and AGC target $=1 \mathrm{e}^{5}$ or $2 \mathrm{e}^{5}$. LC-HRMS was run once per sample. QC samples were measured after every five samples in order to normalize peak areas and pick stable peaks.

\section{Data processing and analysis}

Almost all data (excluding one SAD sample) were processed with Compound Discoverer 2.1 (Thermo Fisher Scientific). For aligning retention times, the following parameters were used: alignment model=adaptive curve; maximum shift $[\mathrm{min}]=0.2$; mass tolerance $[\mathrm{ppm}]=5$. For detecting unknown compounds, the following parameters were used: mass tolerance $[\mathrm{ppm}]=5$; intensity tolerance $[\%]=30$, minimum peak intensity $=1,000,000$. Peaks in which the QC sample coverage was less than $50 \%$ and relative standard deviation of the areas under the peaks was more than $20 \%$ were excluded.

The area under each peak was corrected linearly using the area under the peak of the QC samples to eliminate drift in the signal for each metabolic feature in the samples before multivariate and statistical analysis. Principal component analysis (PCA) was performed using normalized areas under the peaks with Compound Discoverer 2.1. To detect differential metabolites between the resting state and SAD groups, volcano plot analysis by Student's t-test was performed with Compound Discoverer 2.1. Adjusted $P$-values were calculated using the Benjamini-Hochberg algorithm, while keeping in mind the false discovery rate. The $\mathrm{X}$-axis represents $\log 2$ fold change ( $\mathrm{SAD} /$ resting state), and the $\mathrm{Y}$-axis represents $-\log 10$ adjusted $P$-value.

\section{Peak identification}

KEGG numbers for each peak were obtained from the KEGG COMPOUND Database (https://www.genome. $\mathrm{jp} / \mathrm{kegg} /$ compound/) using in-house software written in Python. This software sent accurate masses of each peak to the KEGG database and in turn received KEGG numbers for compounds with exactly the same masses. Mass tolerance was $5 \mathrm{ppm}$. MS/MS spectra of these metabolites were compared with those in the mzCloud database or in published papers in order to conclusively identify them.

\section{Metabolite Set Enrichment Analysis (MSEA) and pathway analysis}

MSEA was performed with MetaboAnalyst (http://www. metaboanalyst.ca) using the KEGG number of each peak. Pathway analysis was performed on KEGG metabolic pathways in Equus Caballus with the user data mapping tool in the KEGG database.

\section{Results}

\section{Peaks detected by MS}

One SAD sample was excluded due to poor measurement data. After data processing, 5,010 peaks were detected in horse plasma samples by LC-HRMS. Based on their accurately determined molecular masses, these peaks were searched in the KEGG metabolite database. As a result, 1,256 of 5,010 peaks (approximately $25 \%$ ) were annotated with KEGG numbers (i.e., the IDs of the compounds); however, there were some peaks that were annotated with multiple KEGG numbers. The remaining peaks (approximately $75 \%$ ) were not annotated with KEGG numbers because their masses were not contained in the KEGG database.

\section{$P C A$}

PCA was carried out using all detected peaks $(5,010)$. As shown in Fig. 1, 30 resting state and 29 SAD samples were clearly divided into two different groups. The first principal component (X-axis) divided the samples into the two groups, and the second principal component (Y-axis)

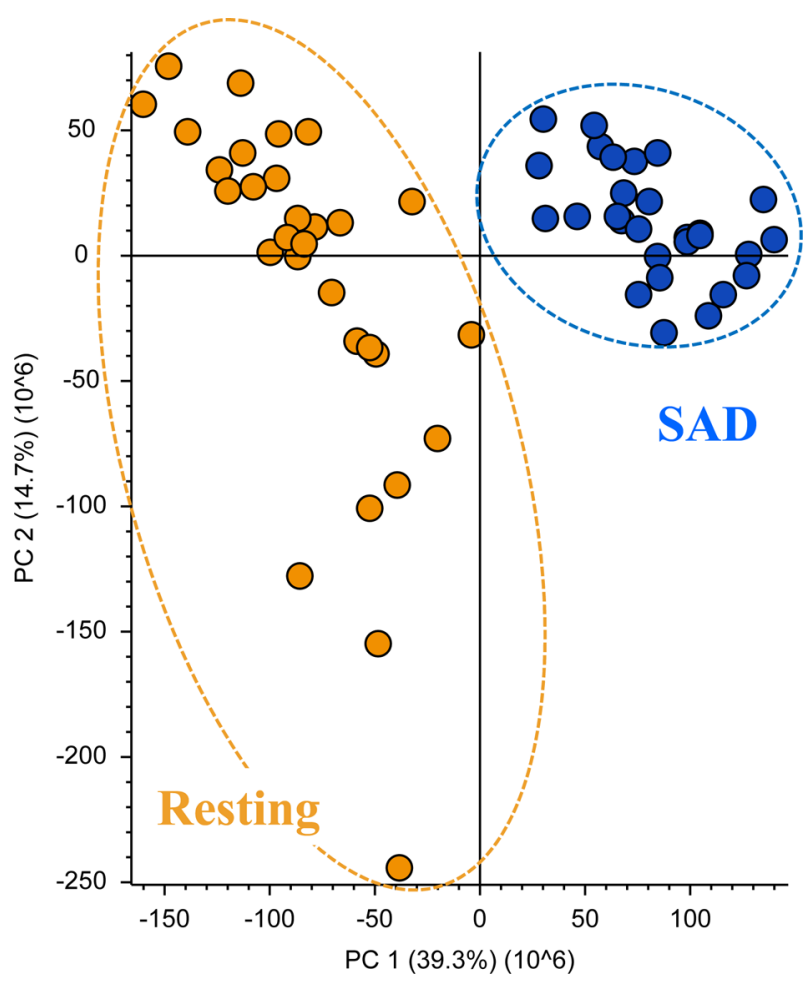

Fig. 1. Principal component analysis using all detected peaks. Orange and blue dots indicate resting state and stage after racing for doping test (SAD) samples, respectively. The X-axis represents the first principal component, and the Y-axis represents the second principal component. 


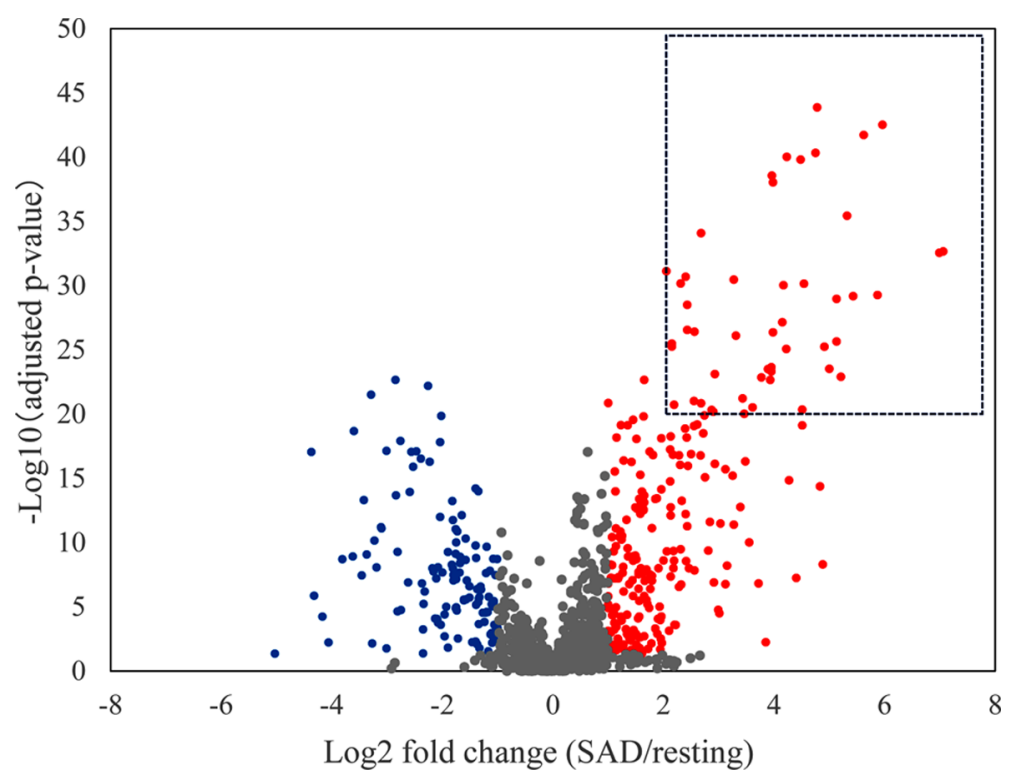

Fig. 2. Volcano plot analysis for identifying metabolites differentially detected in horse plasma between the resting state and stage after racing for doping test (SAD) groups. Red and blue dots indicate characteristic metabolites in the SAD and resting state groups (adjusted $P$-value $<0.05$, fold change $>2$ times), respectively. Grey dots indicate metabolites that are not different between the two groups. Red dots present within the square represent metabolites that are the most characteristic of SAD samples (adjusted $P$-value $<1.0 \times 10^{-20}$, fold change $>4$ times).

explained the individual variations of samples in the resting state group.

\section{Volcano plot analysis}

Volcano plot analysis was performed for the 1,256 KEGG annotated metabolites (Fig. 2). This analysis revealed that the levels of 247 metabolites (red circles in Fig. 2) were significantly increased in the SAD group (adjusted $P$-value $<0.05$, fold change $>2$ times), while the levels of 125 metabolites (blue circles in Fig. 2) were significantly decreased in the SAD group (adjusted $P$-value $<0.05$, fold change $>2$ times). In particular, 49 of the 247 metabolites in the SAD group (dotted square in Fig. 2) showed a significant increase in levels (adjusted $P$-value $<1.0 \times 10^{-20}$, fold change $>4$ times); except for three metabolites, a similar increase in metabolite levels was not observed in the resting state group. In addition, the remaining 884 metabolites were not significantly different between the two groups.

\section{MSEA and pathway analysis}

MSEA was performed on metabolites whose levels were altered in the SAD group. However, significantly different pathways $(P$-value $<0.05)$ between the two groups were not identified. Seven of the 49 metabolites observed in the SAD group were intensively mapped to inosine, xanthosine, uric acid, allantoin, hypoxanthine, xanthine, and deoxyinosine positions in part of the purine metabolism pathway
(Fig. 3). Inosine, xanthosine, uric acid, and allantoin were conclusively identified based on the MS/MS data, which were compared with the mzCloud database or published papers [1]. However, hypoxanthine and xanthine were not identified because they were produced by in-source fragmentation of inosine and xanthosine. Deoxyinosine was also not identified because the MS/MS data were different from those in mzCloud.

Areas under the peaks of the four identified metabolites were compared between the SAD and resting state groups (Fig. 4). The levels of all four metabolites significantly increased in the resting state group. The fold changes of the peak areas of xanthosine, inosine, uric acid, and allantoin were $127,35,6.4$, and 7.6 , respectively.

\section{Discussion}

In this study, 5,010 peaks were detected from horse plasma, and approximately $25 \%$ of these were annotated by KEGG analysis. The remaining peaks (approximately 75\%) may not have been annotated due to fragmented metabolites or due to metabolites having complex structures [13, 14]. Although there were many peaks that were not annotated by KEGG, PCA of the 5,010 peaks revealed that all samples were clearly divided into two groups-SAD and resting state. This suggested that detected peaks, including nonannotated ones, reflect the metabolic differences between 


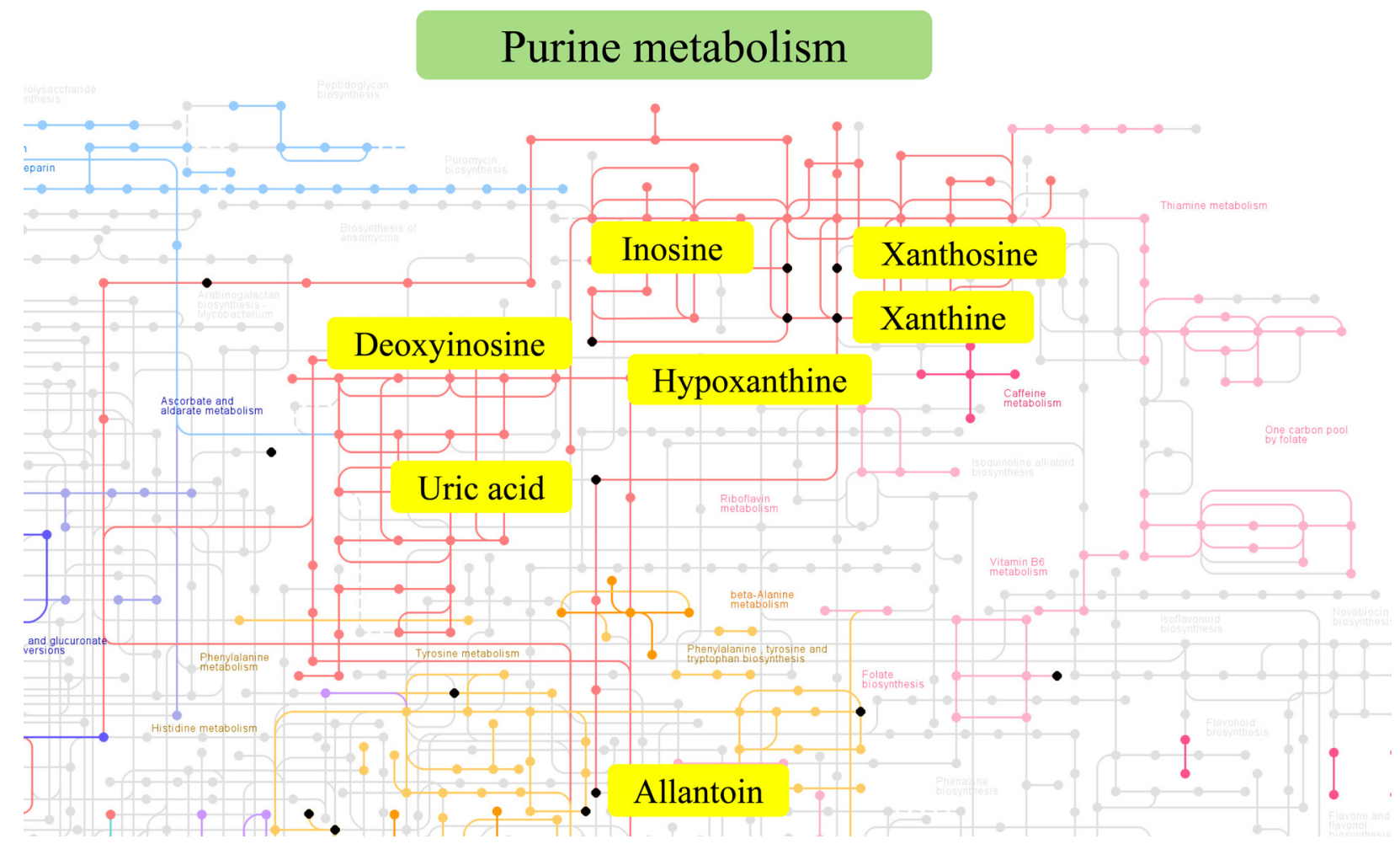

Fig. 3. KEGG pathway analysis focused on purine metabolism pathway. Black dots indicate metabolites that are observed in stage after racing for doping test (SAD) group. Seven metabolites, inosine, xanthosine, uric acid, allantoin, hypoxanthine, xanthine, and deoxyinosine, were intensively mapped to the purine metabolism pathway.
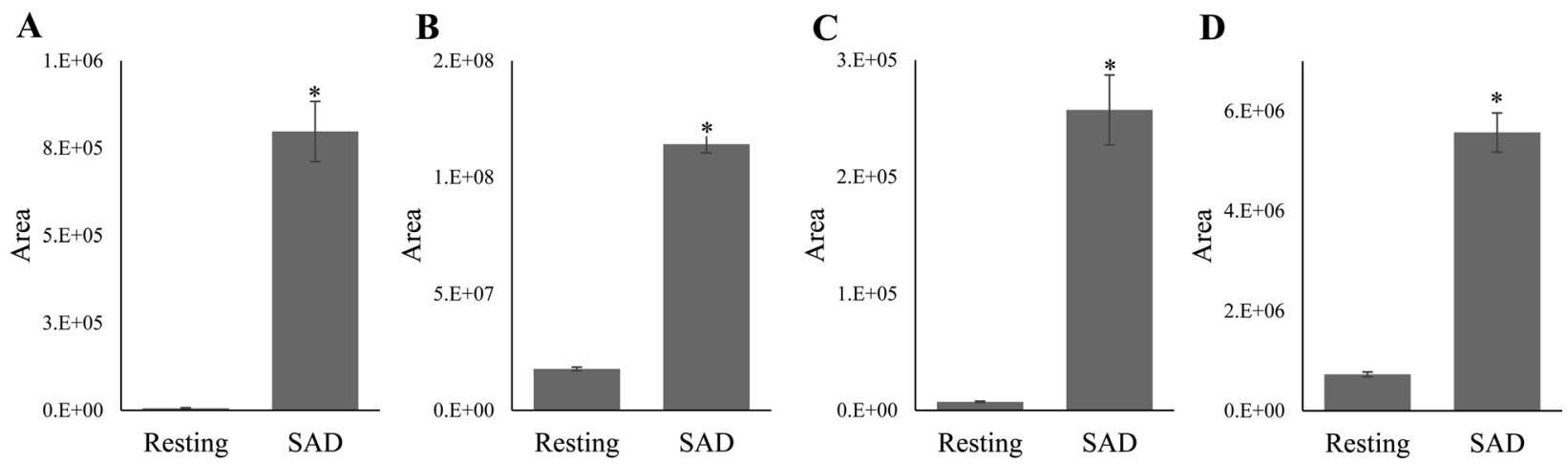

Fig. 4. Quantitative differences in the metabolites identified in the purine metabolism pathway between the resting state and stage after racing for doping test (SAD) groups. A) Xanthosine, B) uric acid, C) inosine, D) allantoin. *Adjusted $P$-value $<0.05$.

SAD and the resting state.

Peak identification was performed using only the accurate mass of each peak; therefore, some peaks had multiple KEGG numbers. This may be the reason why we could not identify any pathway that was significantly different between SAD and the resting state.

Interestingly, a metabolic increase (adjusted $P$-value $<1.0 \times 10^{-20}$, fold change $>4$ times) was observed in the
SAD group. The identified metabolites were specifically mapped to the purine metabolism pathway. These finding indicated that a lot of adenosine triphosphate (ATP) was consumed during extensive exercise [18, 19]. Therefore, these metabolites are not suitable as doping biomarkers but may be used as biomarkers for extensive exercise.

Via the purine pathway, ATP is metabolized to allantoin which is then excreted in urine. In this study, the fold 
changes of metabolites (i.e., inosine and xanthosine) that were located upstream of the purine pathway were much higher when compared with those (i.e., uric acid and allantoin) that were located downstream. These differences may be due to the degradation of ATP at several stages.

When using metabolomics approaches to identify biomarkers for the detecting the use of prohibited substances, animals need to be maintained under controlled conditions, usually resting conditions, to eliminate other factors. As our results have revealed that multiple metabolites were altered in the SAD group, metabolites that are not altered in the SAD group should be selected as biomarkers for detecting doping to improve specificity.

In addition, the samples from SAD were tightly clustered, while samples from the resting state were broadly distributed. Metabolites under resting conditions were diverse and altered with each horse. Therefore, metabolites used as biomarkers should be first quantified and evaluated using multiple samples from different horses.

This was a preliminary study that comprehensively compared metabolites in horse plasma samples taken under resting conditions and after racing, and its aim was to identify biomarkers for doping in horses. We expect that metabolomics approaches will contribute to the detection of prohibited substances which cannot be detected by conventional methods, such as unexpected genes and unknown designer drugs, ultimately ensuring fairness in horse racing.

\section{Acknowledgments}

This study was partially supported by a Grant-in-Aid from the Japan Racing Association (2017-2019). We also thank the Japan Racing Association for providing the experimental samples.

\section{References}

1. Berthemy, A., Newton, J., Wu, D., and Buhrman, D. 1999. Quantitative determination of an extremely polar compound allantoin in human urine by LC-MS/MS based on the separation on a polymeric amino column. J. Pharm. Biomed. Anal. 19: 429-434. [Medline] [CrossRef]

2. Dunn, W.B., Broadhurst, D., Begley, P., Zelena, E., Francis-McIntyre, S., Anderson, N., Brown, M., Knowles, J.D., Halsall, A., Haselden, J.N., Nicholls, A.W., Wilson, I.D., Kell, D.B., Goodacre, R., Human Serum Metabolome (HUSERMET) Consortium 2011. Procedures for largescale metabolic profiling of serum and plasma using gas chromatography and liquid chromatography coupled to mass spectrometry. Nat. Protoc. 6: 1060-1083. [Medline] [CrossRef]

3. González-Domínguez, R., García-Barrera, T., and GómezAriza, J.L. 2014. Combination of metabolomic and phos- pholipid-profiling approaches for the study of Alzheimer's disease. J. Proteomics 104: 37-47. [Medline] [CrossRef]

4. Guan, F., and Robinson, M.A. 2017. Comprehensive solidphase extraction of multitudinous bioactive peptides from equine plasma and urine for doping detection. Anal. Chim. Acta 985: 79-90. [Medline] [CrossRef]

5. Guddat, S., Solymos, E., Orlovius, A., Thomas, A., Sigmund, G., Geyer, H., Thevis, M., and Schänzer, W. 2011. High-throughput screening for various classes of doping agents using a new 'dilute-and-shoot' liquid chromatography-tandem mass spectrometry multi-target approach. Drug Test. Anal. 3: 836-850. [Medline] [CrossRef]

6. Ho, E.N.M., Chan, G.H.M., Wan, T.S.M., Curl, P., Riggs, C.M., Hurley, M.J., and Sykes, D. 2015. Controlling the misuse of cobalt in horses. Drug Test. Anal. 7: 21-30. [Medline] [CrossRef]

7. Ho, E.N.M., Kwok, W.H., Lau, M.Y., Wong, A.S.Y., Lam, K.K.H., Stewart, B.D., and Wan, T.S.M. 2014. Doping control analysis of filgrastim in equine plasma and its application to a co-administration study of filgrastim and recombinant human erythropoietin in the horse. J. Chromatogr. A 1338: 92-101. [Medline] [CrossRef]

8. Jandrić, Z., Roberts, D., Rathor, M.N., Abrahim, A., Islam, M., and Cannavan, A. 2014. Assessment of fruit juice authenticity using UPLC-QToF MS: a metabolomics approach. Food Chem. 148: 7-17. [Medline] [CrossRef]

9. Jobard, E., Pontoizeau, C., Blaise, B.J., Bachelot, T., Elena-Herrmann, B., and Trédan, O. 2014. A serum nuclear magnetic resonance-based metabolomic signature of advanced metastatic human breast cancer. Cancer Lett. 343: 33-41. [Medline] [CrossRef]

10. Joré, C., Loup, B., Garcia, P., Paris, A.C., Popot, M.A., Audran, M., Bonnaire, Y., Varlet-Marie, E., and BaillyChouriberry, L. 2017. Liquid chromatography - high resolution mass spectrometry-based metabolomic approach for the detection of Continuous Erythropoiesis Receptor Activator effects in horse doping control. J. Chromatogr. A 1521: 90-99. [Medline] [CrossRef]

11. Lee, J.E., Lee, B.J., Chung, J.O., Kim, H.N., Kim, E.H., Jung, S., Lee, H., Lee, S.J., and Hong, Y.S. 2015. Metabolomic unveiling of a diverse range of green tea (Camellia sinensis) metabolites dependent on geography. Food Chem. 174: 452-459. [Medline] [CrossRef]

12. Lopez-Sanchez, P., de Vos, R.C.H., Jonker, H.H., Mumm, R., Hall, R.D., Bialek, L., Leenman, R., Strassburg, K., Vreeken, R., Hankemeier, T., Schumm, S., and van Duynhoven, J. 2015. Comprehensive metabolomics to evaluate the impact of industrial processing on the phytochemical composition of vegetable purees. Food Chem. 168: 348-355. [Medline] [CrossRef]

13. Mahieu, N.G., and Patti, G.J. 2017. Systems-level annotation of a metabolomics data set reduces 25000 features to fewer than 1000 unique metabolites. Anal. Chem. 89: 10397-10406. [Medline] [CrossRef] 
14. Mahieu, N.G., Spalding, J.L., Gelman, S.J., and Patti, G.J. 2016. Defining and detecting complex peak relationships in mass spectral data: the Mz.unity algorithm. Anal. Chem. 88: 9037-9046. [Medline] [CrossRef]

15. Moulard, Y., Bailly-Chouriberry, L., Boyer, S., Garcia, P., Popot, M.A., and Bonnaire, Y. 2011. Use of benchtop exactive high resolution and high mass accuracy orbitrap mass spectrometer for screening in horse doping control. Anal. Chim. Acta 700: 126-136. [Medline] [CrossRef]

16. Raro, M., Ibáñez, M., Gil, R., Fabregat, A., Tudela, E., Deventer, K., Ventura, R., Segura, J., Marcos, J., Kotronoulas, A., Joglar, J., Farré, M., Yang, S., Xing, Y., Van Eenoo, P., Pitarch, E., Hernández, F., Sancho, J.V., and Pozo, O.J. 2015. Untargeted metabolomics in doping control: detection of new markers of testosterone misuse by ultrahigh performance liquid chromatography coupled to high-resolution mass spectrometry. Anal. Chem. 87: 8373-8380. [Medline] [CrossRef]

17. Shima, N., Miyawaki, I., Bando, K., Horie, H., Zaitsu, K., Katagi, M., Bamba, T., Tsuchihashi, H., and Fukusaki, E. 2011. Influences of methamphetamine-induced acute intoxication on urinary and plasma metabolic profiles in the rat. Toxicology 287: 29-37. [Medline] [CrossRef]

18. Stathis, C.G., Zhao, S., Carey, M.F., and Snow, R.J. 1999. Purine loss after repeated sprint bouts in humans. J. Appl. Physiol. 87: 2037-2042. [Medline] [CrossRef]
19. Stefano, B., Franco, T., and Oberosler, R. 1999. Plasma lactate and purine derivatives accumulation after exercise of increasing intensity in standardbred horses. J. Equine Vet. Sci. 19: 463-468. [CrossRef]

20. Tozaki, T., Gamo, S., Takasu, M., Kikuchi, M., Kakoi, H., Hirota, K.I., Kusano, K., and Nagata, S.I. 2018. Digital PCR detection of plasmid DNA administered to the skeletal muscle of a microminipig: a model case study for gene doping detection. BMC Res. Notes 11: 708. [Medline] [CrossRef]

21. Tozaki, T., Ohnuma, A., Takasu, M., Kikuchi, M., Kakoi, H., Hirota, K.I., Kusano, K., and Nagata, S.I. 2019. Droplet Digital PCR Detection of the Erythropoietin Transgene from Horse Plasma and Urine for Gene-Doping Control. Genes (Basel) 10: 243. [Medline] [CrossRef]

22. Wang, Y., Caldwell, R., Cowan, D.A., and Legido-Quigley, C. 2016. LC-MS-based metabolomics discovers purine endogenous associations with low-dose salbutamol in urine collected for antidoping tests. Anal. Chem. 88: 2243-2249. [Medline] [CrossRef]

23. Wong, K.S., Chan, G.H.M., Ho, E.N.M., and Wan, T.S.M. 2016. Simultaneous detection of recombinant growth hormones in equine plasma by liquid chromatography/highresolution tandem mass spectrometry for doping control. J. Chromatogr. A 1478: 35-42. [Medline] [CrossRef] 